\title{
Magnetic resonance imaging findings of a patient with bilateral submandibular gland aplasia
}

\author{
Ender Alkan ${ }^{1}$, Turgay Kara ${ }^{2}$, Zeynep Öztürk İnal ${ }^{3}$ \\ ${ }^{I}$ Department of Radiology, Aksaray University School of Medicine, Aksaray, Turkey \\ ${ }^{2}$ Department of Radiology, Konya Training and Research Hospital, Konya, Turkey \\ ${ }^{3}$ Department of Obstetrics and Gynecology, Konya Training and Research Hospital, Konya, Turkey
}

\begin{abstract}
Aplasia of the major salivary glands are very rare, and commonly occurs at the parotid gland. Patients are usually asymptomatic and diagnosed incidentally. But dry mouth, difficulty in swallowing, dental problems may be seen. Also mass complaints may occur due to compensatory hypertrophy of the other major salivary glands. Herein, we presented the magnetic resonance imaging findings of a very rare case of bilateral submandibular gland aplasia with compensatory hypertrophy of sublingual glands.
\end{abstract}

Eur Res J 2018;4(2):126-128

Keywords: salivary glands, magnetic resonance imaging, compensatory hypertrophy, submandibular gland aplasia

\section{Introduction}

Aplasia of the major salivary glands is a very rare situation of which the incidence is not accurately known. Approximately 40 cases with congenital absence of the submandibular gland have been reported in the literature [1]. Although the etiology is not proven, it has been thought to occur due to a defect in fetal development [2]. In addition; submandibular gland aplasia can be seen in Lacrimo-aurikulo-dentodigital syndrome (LADD), Mandibulofacial Dysosteosis (Treacher- Collins Syndrome), Ectodermal Dysplasia with lacrimal-thyroid aplasia or dysplasia [3, 4].
In this article, we presented magnetic resonance imaging (MRI) findings of bilateral submandibular gland aplasia with compensatory hypertrophy of sublingual glands in a 21-year-old woman.

\section{Case Presentation}

A 21-year-old woman referred to ear-nose and throat clinic with long-term xerostomia and teeth complaints. In physical examination, a solid-mobile mass in submandibular region was found by palpation. There was no significant data in laboratory, so an 


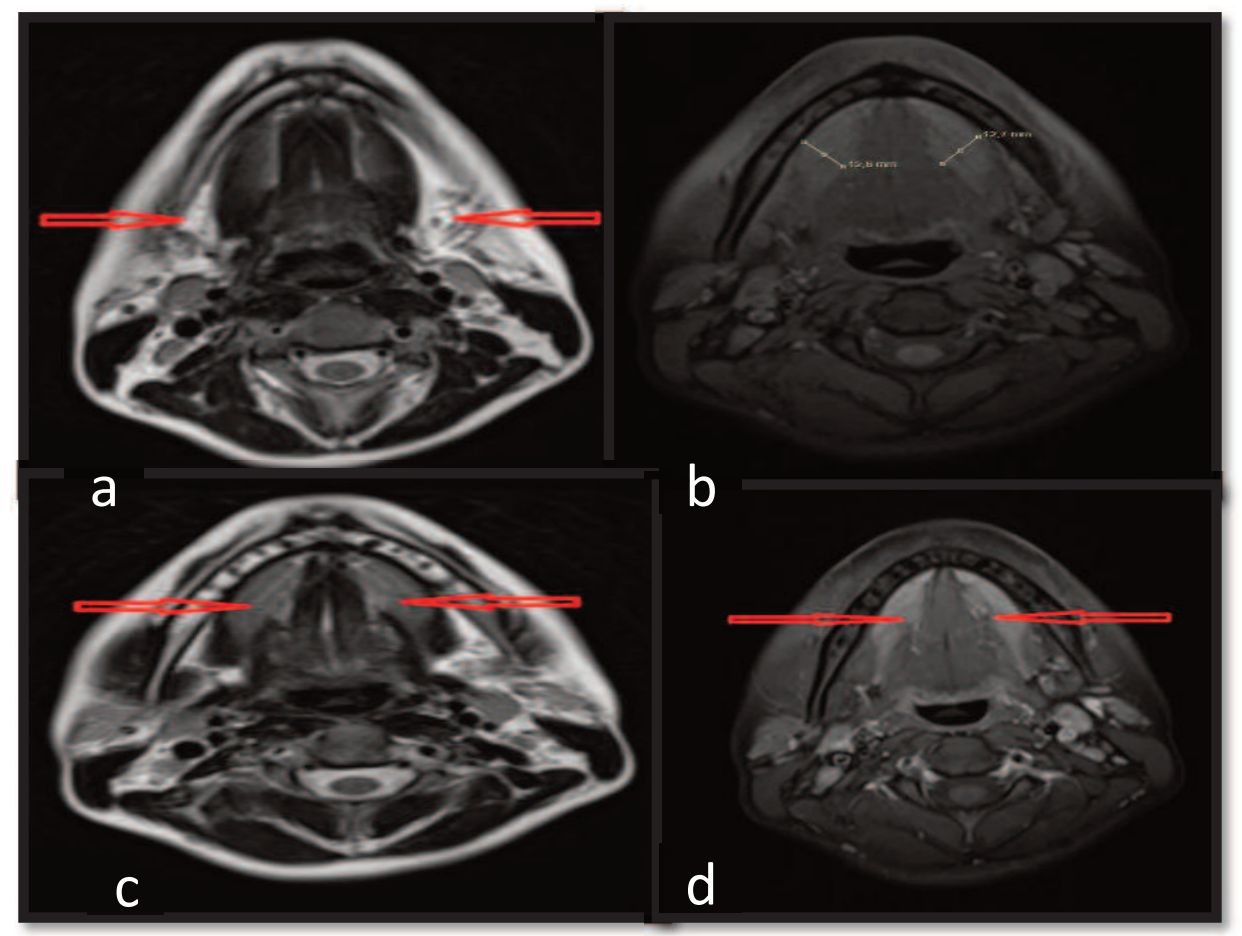

Figure 1. (a) Both bilateral submandibular glands were not seen in their normal localization- T2A view (arrows), (b) Sublingual gland thickness $12.8 \mathrm{~mm}$ in right and $12.7 \mathrm{~mm}$ in left- T1A view, (c) Sublingual glands T2A view and (d) Sublingual glands T2A contrasted fat saturation view (bidirectional arrows).

ultrasound screening was planned. Absence of submandibular glands and hypertrophy of sublingual glands were detected by ultrasound.

After that a contrasted neck MRI were applied and no submandibular gland were seen in normal region either (Figure 1a). Besides we measured the thickness of sublingual glands $12.8 \mathrm{~mm}$ in right, and $12.7 \mathrm{~mm}$ in left (Figures 1b, 1c and 1d). Whereas Sumi et al. [5] reported normal thickness of those as $5 \mathrm{~mm}$ or less. Finally, the patient was diagnosed as bilateral submandibular gland aplasia with compensatory hypertrophy of sublingual glands.

\section{Discussion}

Aplasia of salivary glands is observed rarely, and the reported number is about 40 cases [1]. Aplasia of submandibular glands may be unilateral or bilateral, total or partial [6]. The etiology is claimed to be a defect of major salivary gland and oral ectoderm proliferation and migration between 4-8 weeks during fetal development. It can be associated with 1 st and 2nd branchial arch anomalies. Submandibular glands maturate after parotid gland. The maturation of sublingual and minor salivary glands is later [7].

Half of the cases are asymptomatic and may be detected incidentally. Xerostomia, dysphagia and tooth problems are major complaints in symptomatic patients. In some patients, other salivary glands may have a compensatory hypertrophy as the case presented in this report, and they can be evaluated as mass [1].

Anamnesis and bimanual palpation are very important. Ultrasound, computed tomography, MRI, sialography, even scintigraphy can be applied for diagnosis [8]. By these imaging methods, absence of submandibular glands has to be proven and associated sublingual gland hypertrophy may be observed; the tumors originating from the floor of mouth should to be excluded. Radiologists must be familiar to this benign situation to prevent unnecessary biopsies. Clinicians must be very careful about branchial arc anomalies associated to major salivary gland aplasia. Sjogren syndrome should be considered in the differential diagnosis, and it can be excluded if the submandibular glands are detected in normal localization. The tumors of mouth floor also must be take into account. They are mostly diagnosed by imaging; however, biopsy and pathological evaluation should be performed in some cases [9].

\section{Conclusion}

Finally, major salivary gland aplasia and especially bilateral submandibular gland aplasia should be kept in mind in patients with the symptoms 
such as neckmass, teeth problems, dysphagia and mouth dryness.

\section{Informed consent}

Written informed consent was obtained from the patient for the publication of this case report.

\section{Conflict of interest}

The authors declared that there are no potential conflicts of interest with respect to the research, authorship, and/or publication of this article.

\section{References}

[1] Kara M, Güçlü O, Dereköy FS, Resorlu M, Adam G. Agenesis of submandibular glands: a report of two cases with review of literature. Case Rep Otolaryngol 2014;2014:569026.

[2] Mathison CC, Hudgins PA. Bilateral submandibular gland aplasia with hypertrophy of sublingual glands. Otolaryngol Head Neck Surg
2008;138:119-20.

[3] Inan UU, Yilmaz MD, Demir Y, Degirmenci B, Ermis SS, Ozturk F. Characteristics of lacrimo-auriculo-dento-digital (LADD) syndrome: case report of a family and literature review. Int $\mathrm{J}$ Pediatr Otorhinolaryngol 2006;70:1307-14.

[4] Singh P, Warnakulasuriya S. Aplasia of submandibular salivary glands associated with ectodermal dysplasia. J Oral Pathol Med 2004;33:634-6.

[5] Sumi M, Izumi M, Yonetsu K, Nakamura T. Sublingual gland: MR features of normal and diseased states. AJR Am J Roentgenol 1999;172:717-22.

[6] Ferreira AP, Gomez RS, Castro WH, Calixto NS, Silva RA, Aguiar MJ. Congenital absence of lacrimal puncta and salivary glands: report of a Brazilian family and review. Am J Med Genet 2000;94:32-4.

[7] Strong BE, Johns ME. Anatomy and physiology of the salivary glands. In: Bailey B, Newlands SD, editors. Head and neck surgeryotolaryngology. Philadelphia: Lippincott, Williams and Wilkins, 2006:517-21.

[8] Roh JL. Unilateral submandibular gland aplasia: an isolated phenomenon of early fetal development. Otolaryngol Head Neck Surg 2006;135:332-4.

[9] Eleftheriadis I, Papadimitriou P, Tzelepi H. [Submandibular swelling and its differential diagnosis]. Hell Period Stomat Gnathopathoprosopike Cheir 1990;5:59-68. [Article in Greek, Modern) 